\title{
Saúde Mental de Alunos de Medicina Submetidos à Aprendizagem Baseada em Problemas: Revisão Sistemática da Literatura
}

\section{Mental Health of Medical Students Submitted to Problem-Based Learning: Systematic Literature Review}

\author{
Carlos Emanoel Chaves da Silva ${ }^{I}(1)$ \\ Alef Oliveira do Nascimento ${ }^{I}$ (i) \\ Israel Junior Borges do Nascimento ${ }^{I I}$ (D) \\ Mayra Hermínia Simões Hamad Farias do Couto ${ }^{I I I}[\mathbb{D}$ \\ Luiz Euclides Coelho de Souza Filho ${ }^{I V}$ (D) \\ Katiane da Costa Cunha ${ }^{I}$ (D)
}

\section{PALAVRAS-CHAVE}

- Saúde Mental.

- Aprendizagem Baseada em Problemas.

- Estudantes de Medicina.

- Educação em Saúde.

- Educação Médica.
REVISTA BRASILEIRA DE EDUCAÇÃO MÉDICA

44 (4) : e115; 2020
${ }^{\mathrm{I}}$ Universidade do Estado do Pará, Marabá, Pará, Brasil.

${ }^{\mathrm{II}}$ Universidade Federal de Minas Gerais, Belo Horizonte, Minas Gerais, Brasil.

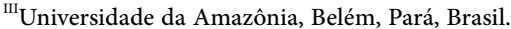

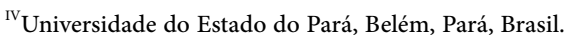




\section{KEYWORDS}

- Mental Health.

- Problem-Based Learning.

- Students Medical.

- Health Education.

-MedicalEducation.

Recebido em 11/3/20

Aceito em 16/7/20

\section{INTRODUÇÃO}

A saúde mental consiste no bem-estar psicológico ou ajustamento adequado, em particular quando tal ajustamento está de acordo com os padrões de relações humanas aceitos pela comunidade. Algumas características de saúde mental são: razoável independência, autoconfiança, auto-orientação, capacidade de fazer um trabalho, capacidade de assumir responsabilidade e fazer esforços necessários, confiabilidade, persistência, capacidade de relacionar-se e trabalhar com outras pessoas, cooperação, capacidade de trabalhar sob autoridade, entre outras ${ }^{1}$.

Apesar do caráter subjetivo e transcultural da saúde mental ${ }^{2}$, um aspecto imutável é o fato de ela ir além da simples ausência de perturbações psicológicas. Nesse sentido, diversas síndromes e variados transtornos mentais podem ser classificados em diferentes níveis, desde os mais graves até os mais brandos, de modo que a avaliação deve ser multiaxial considerar todos os aspectos de saúde e situação social do indivíduo $^{3}$. Ademais, o adoecimento mental promove consequências para diversos âmbitos da vida: individual (baixa autoestima, dificuldade de concentração, distúrbios do sono, entre outros), ocupacional, organizacional (absenteísmo e presenteísmo) e social ${ }^{4}$.

Quando se observa a relação dos determinantes sociais no processo de adoecimento psíquico, tem-se como ponto inicial a relação de causalidade desses transtornos com a fase de formação e atuação profissional. Alguns cursos universitários, bem como determinadas atividades laborais, podem desencadear ou agravar esse processo, a exemplo do curso de Medicina, tornando-se uma das preocupações de instituições como a Organização Mundial da Saúde (OMS),Associação Brasileira de Educação Médica (Abem) e Organização Pan-Americana de Saúde (Opas) ${ }^{5}$. Isso ocorre, entre outros fatores, pelo fato de o curso de Medicina exigir grande dedicação, esforço, sacrifício e resistência física e emocional

\section{ABSTRACT}

Introduction: Medical students are subjected to potentially stressful factors that can lead to psychological illness. Alternatives to traditional training methods, such as Problem-Based Learning (PBL), can impact on students' mental health. The aim of this study was to carry out a systematic review of studies that evaluate the mental health of medical students submitted to the Problem-Based Learning (PBL) method. Method: A highly sensitive search was carried out in the main scientific databases associated with mental health (Cochrane Library, MEDLINE via PUBMED, Regional Health Portal (VHL), Eric and EMBASE) with inclusion up to September 22, 2019. Indexed descriptions and identification terms were defined by specialists and evaluated by the information technology group of the StateUniversity of Pará. The inclusion criteria for the study search were: articles presented in full, written in any language, published in the period from 1998 to 2018, focusing on the mental health of medical students submitted to the PBLmethod. Articles that did not assess menta articles in which the sample was not submitted to $P B L$, andreview articleswere excluded. After selection, ROBINS-I tool. Results: 1261 studies were identified, of which 1251 were excluded following the PRISMA as stimulating practical activities, a fact that generates a certain degree of satisfaction. Conclusion: Medical students submitted to the PBL method are more likely to be associated with better learning outcomes and medical-scientific performance. However, better study designs, based on rigorous systematic methodologies are required in order to elucidate the benefits and harms of this educational methodology. 
Considerando os potenciais riscos de adoecimento mental de discentes em cursos de nível superior em saúde ${ }^{11,12,13,14}$, principalmente de Medicina durante o percurso curricular, metodologias como a $\mathrm{ABP}$ tornam-se fundamentais como método alternativo ao sistema tradicional unilateral e verticalizado. Nos últimos anos, diversos estudos têm evidenciado os potenciais benefícios da $A B P$ na saúde mental de estudantes de Medicina. Entretanto, mesmo com a crescente produção de literatura científica acerca dessa metodologia, nenhum estudo de qualidade de evidência e efetividade dessa intervenção foi identificado até o momento. Dessa forma, o objetivo do presente estudo foi realizar uma revisão sistemática de estudos que avaliaram a saúde mental de estudantes de Medicina submetidos ao método ABP.

\section{MÉTODOS}

O protocolo de execução desta revisão sistemática foi publicado na Plataforma Prospero (CRD42020152445), e todo o percurso metodológico dela está em consonância com premissas do Preferred Reporting Items for Systematic Reviews and Meta-Analyses (Prisma) ${ }^{15}$.

\section{Fontes de dados e pesquisa}

Uma busca de alta sensibilidade foi realizada em vários bancos de dados eletrônicos para identificar todos os estudos relevantes que avaliaram a saúde mental de alunos do curso de Medicina submetidos ao método ABP. As bases de dados foram: Cochrane Library, Medline via PubMed, Portal Regional da Biblioteca Virtual em Saúde (BVS), Eric e Embase.

\section{Critérios de inclusão e exclusão de estudos}

Incluíram-se ensaios clínicos randomizados e não randomizados, assim como estudos observacionais, que apresentassem de forma integral seus respectivos conteúdos. Os critérios de elegibilidade foram estudos que avaliaram a saúde mental em discentes de Medicina submetidos ao método $\mathrm{ABP}$ e que os compararam com métodos tradicionais ou quaisquer outros métodos de aprendizagem. Definiram-se como método ABP estudos com abordagens pedagógicas capazes de desenvolver a habilidade de "aprender a aprender", em que o discente seja desafiado a agir no intuito de transpor um obstáculo e realizar uma aprendizagem por meio da resolução de uma situação-problema com recorte de uma realidade, de um cenário complexo, de algo que é dinâmico ${ }^{9}$, assim como as metodologias que abrangeram sessões tutoriais, atividades em laboratórios, apresentações científicas e o uso de filmes ${ }^{10}$. Não houve restrição idiomática nem temporal quanto aos estudos obtidos. Todos os estudos que não obedeceram a esses critérios de inclusão foram excluídos, como os artigos que não avaliaram a saúde mental, aquelesem que a amostra não foi submetida à metodologia de ensino ABP,assim como artigos de revisão literária, relatos de caso e caso de séries.

Extração de dados e síntese da dados

Exportaram-se as referências recuperadas nas estratégias de pesquisa para um arquivo Mendeley, e os resultados foram resumidos no diagrama de fluxo Prisma (Figura 1). Dois pesquisadores independentes (KCC e MR) selecionaram títulos e resumos, e os textos completos em potencial

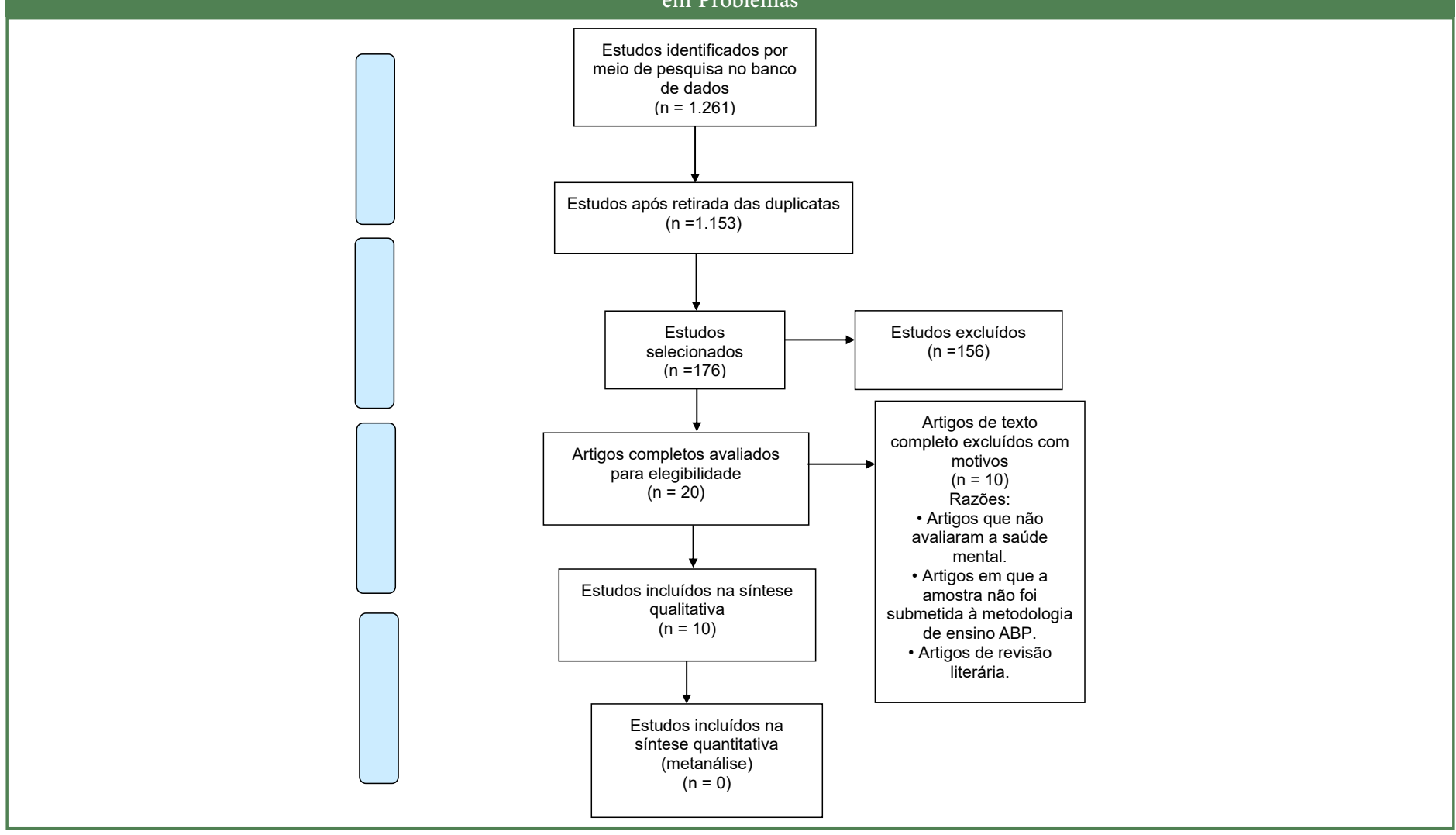

Fonte: Elaborada pelos autores.

\footnotetext{
$3 \mid$\begin{tabular}{l|l} 
REVISTA BRASILEIRA DE EDUCAÇÃO MÉDICA \\
\hline 44 (4) : el15; 2020
\end{tabular}
} 
foram avaliados quanto aos critérios de elegibilidade. Nos casos em que houve desacordos, um terceiro pesquisador independente (MH) resolveu as discrepâncias. Na revisão sistemática, adotaram-se os seguintes tipos de estudo: observacional, transversal e longitudinal.

Para extração dos dados, dois pesquisadores independentes (KCC e MR) utilizaram um formulário preestabelecido para a coleta das informações sobre as características dos estudos: dados dos estudos (autores, nome do periódico, país e cenário do estudo, ano de publicação), dados sociodemográficos dos alunos de Medicina (sexo, faixa etária, estado civil, ocupação e renda familiar) e informações metodológicas (objetivo do estudo, desenho, tamanho da amostra total, aspecto ou variável da saúde mental avaliada e instrumentos usados para avaliá-la).

Inicialmente, havia a expectativa de realizar a síntese da dados a partir de dados numéricos, de forma dicotômica ou contínua. Entretanto, após a constatação de que havia uma ampla descrição dos resultados dos estudos selecionados, percebeu-se a inviabilidade desse critério sintético. Assim, executou-se a sumarização de dados extraídos por meio de uma descrição sumária dos principais achados dos estudos filtrados. Não foi possível realizar a síntese metanalítica em função de contrastes metodológicos nos estudos selecionados.

\section{Avaliação da qualidade}

Todos os estudos incluídos nesta revisão foram avaliados quanto à qualidade de evidência. Para isso, utilizou-se o Risk of Bias in Non-
Randomized Studies-of Interventions (Robins), ferramenta da Cochrane para avaliação da qualidade de estudos observacionais. Tal instrumento avalia estudos de coorte e de caso-controle para revisões sistemáticas de intervenção, além de estimar os riscos de viés desses estudos. Essa ferramenta é composta por sete domínios: os três primeiros (confundimento, seleção dos participantes e aferição da intervenção) podem ser considerados como domínios pré-intervenção, específicos para estudos observacionais; os outros quatro (não recebimento da intervenção, perdas, aferição dos desfechos e relato seletivo dos desfechos) são pós-intervenção, podendo ser também, conceitualmente, potenciais vieses em ensaios clínicos randomizados. Foi elaborado um quadro para o preenchimento das características dos estudos e para a geração do gráfico e sumário de risco de viés. Também seutilizouo Microsoft Excel 2019 para a disposição dos dados em quadros.

\section{RESULTADOS}

Identificaram-se 1.261 estudos por meio de cinco plataformas eletrônicas de dados: 489 referências do Medline, 134 do Embase, 133 do Eric, 382 do Cochrane e 123 do Portal Regional da BVS. Após a exclusão de 108 duplicatas, realizou-se a análise detalhada de 1.153 títulos e resumos. Recuperaram-se artigos em texto completo para os 20 registros restantes, dos quais dezforam excluídos por não avaliarem a saúde mental. Por fim, encontraram-sedez estudos elegíveis para inclusão nesta revisão (Quadro1). A Figura 1 apresenta o diagrama Prisma.

\begin{tabular}{|c|c|c|c|c|c|}
\hline \multicolumn{6}{|c|}{$\begin{array}{l}\text { Quadro } 1 \\
\text { s estudos selecionados }(n=10)\end{array}$} \\
\hline Primeiro autor & País & $\begin{array}{c}\text { Duração do } \\
\text { estudo }\end{array}$ & Desenho do estudo & Variável da saúde mental estudada & $\begin{array}{c}\text { Instrumento utilizado para medir a } \\
\text { variável }\end{array}$ \\
\hline Lewis et al. ${ }^{23}$ & Reino Unido & Não informada & $\begin{array}{l}\text { Observacional e } \\
\text { transversal }\end{array}$ & $\begin{array}{l}\text { Gostar do curso e o estresse relacionado } \\
\text { a ele; insegurança pela expectativa dos } \\
\text { docentes por um currículo confuso; } \\
\text { preocupação com o aprendizado médico; } \\
\text { competição e método educacional; } \\
\text { insuficiência da educação; falta de apoio } \\
\text { docente e tempo para estudos curriculares } \\
\text { e extracurriculares; senso de isolamento } \\
\text { social e anonimato entre os alunos; } \\
\text { conformidade com o esperado do curso }\end{array}$ & $\begin{array}{l}\text { Questionário de autorrelato avaliado pelo } \\
\text { the Perceived Medical Student Stress scale } \\
\text { (PMSS) e Higher Education Stress Inventory } \\
\text { (HESI). }\end{array}$ \\
\hline Barbosa et al. ${ }^{22}$ & Brasil & Não informada & $\begin{array}{l}\text { Observacional e } \\
\text { transversal }\end{array}$ & Burnout & $\begin{array}{l}\text { Questionário abordando as três dimensões } \\
\text { da síndrome de burnout: exaustão } \\
\text { emocional, descrença e eficácia profissional. }\end{array}$ \\
\hline Bahar-ÖzvariŞetal. ${ }^{16}$ & Turquia & Não informada & $\begin{array}{c}\text { Observacional e } \\
\text { longitudinal de caso- } \\
\text { controle }\end{array}$ & $\begin{array}{l}\text { Satisfação com o aprendizado cooperativo } \\
\text { proporcionado pela ABP }\end{array}$ & Questionário sobre satisfação, \\
\hline Lucchetti et al. ${ }^{24}$ & $\begin{array}{l}\text { Brasil e } \\
\text { Estados } \\
\text { Unidos }\end{array}$ & Seis meses & $\begin{array}{l}\text { Observacional, } \\
\text { transversal e } \\
\text { intercultural }\end{array}$ & $\begin{array}{l}\text { Depressão, ansiedade e estresse, empatia, } \\
\text { abertura à espiritualidade, bem-estar, } \\
\text { burnout e qualidade de vida }\end{array}$ & $\begin{array}{l}\text { Escala de Ansiedade e Estresse (DASS-21), } \\
\text { Pesquisa sobre Empathy, Spirituality, and } \\
\text { Wellness in Medicine survey (ESWIM), } \\
\text { Oldenburg Burnout Inventory (Olbi) e The } \\
\text { World Health Organization Quality of Life } \\
\text { (WHOQOL-Bref). }\end{array}$ \\
\hline Trappler $^{17}$ & $\begin{array}{l}\text { Estados } \\
\text { Unidos }\end{array}$ & Não informada & $\begin{array}{l}\text { Observacional e } \\
\text { transversal }\end{array}$ & $\begin{array}{l}\text { Satisfação do aluno com a mudança do } \\
\text { módulo de neurociência para o modelo } \\
\text { ABP }\end{array}$ & Questionário sobre satisfação. \\
\hline
\end{tabular}




\begin{tabular}{|c|c|c|c|c|c|}
\hline \multicolumn{6}{|c|}{ Quadro 1} \\
\hline Primeiro autor & País & $\begin{array}{l}\text { Duração do } \\
\text { estudo }\end{array}$ & Desenho do estudo & Variável da saúde mental estudada & $\begin{array}{c}\text { Instrumento utilizado para medir a } \\
\text { variável }\end{array}$ \\
\hline Yan et al. ${ }^{18}$ & China & Não informada & $\begin{array}{l}\text { Observacional, } \\
\text { transversal e de } \\
\text { coorte }\end{array}$ & Satisfação com a metodologia de ABP & $\begin{array}{l}\text { Questionário de satisfação e exame } \\
\text { avaliativo. }\end{array}$ \\
\hline Holen et al. ${ }^{21}$ & $\begin{array}{l}\text { Estados } \\
\text { Unidos, Nepal } \\
\text { e Noruega }\end{array}$ & Não informada & $\begin{array}{l}\text { Observacional } \\
\text { transversal }\end{array}$ & $\begin{array}{l}\text { Tipo de personalidade relacionado com a } \\
\text { satisfação com o método } \mathrm{ABP}\end{array}$ & $\begin{array}{l}\text { Os traços de personalidade foram aferidos } \\
\text { pela versão curta do NEO Five-Factor } \\
\text { Inventory (NEO-FFI). }\end{array}$ \\
\hline Jiménez-Mejías et al. ${ }^{19}$ & Espanha & Não informada & $\begin{array}{l}\text { Observacional } \\
\text { longitudinal de caso- } \\
\text { controle }\end{array}$ & $\begin{array}{l}\text { Satisfação dos alunos com a } \\
\text { implementação da ABP }\end{array}$ & $\begin{array}{l}\text { Questionário anônimo cujos itens foram } \\
\text { escolhidos entre o Questionário de Avaliação } \\
\text { do Ensino para Participação do Aluno e } \\
\text { o Questionário de Avaliação Didática de } \\
\text { Implicação do Aluno. }\end{array}$ \\
\hline Schauber et al. ${ }^{25}$ & Alemanha & $\begin{array}{l}\text { Vinte e quatro } \\
\text { meses }\end{array}$ & $\begin{array}{l}\text { Observacional, } \\
\text { longitudinal e } \\
\text { prospectivo }\end{array}$ & $\begin{array}{l}\text { Percepção dos alunos sobre o ambiente } \\
\text { de aprendizagem, crenças de autoeficácia, } \\
\text { afeto positivo relacionado ao estudo, } \\
\text { apoio social, indicadores de aprendizado } \\
\text { autorregulado e desempenho acadêmico }\end{array}$ & $\begin{array}{l}\text { Medidas de autorrelato, Lista de Avaliação } \\
\text { de Suporte Interpessoal (adaptada), } \\
\text { Escala Geral de Autoeficácia de Schwarzer } \\
\text { e Jerusalém, Estrutura de Pekrun e } \\
\text { Achievement Emotion Questionnaire. }\end{array}$ \\
\hline Alimoglu et al. ${ }^{20}$ & Turquia & $\begin{array}{l}\text { Vinte e quatro } \\
\text { meses }\end{array}$ & $\begin{array}{l}\text { Observacional, } \\
\text { longitudinal e } \\
\text { prospectivo }\end{array}$ & Satisfação com a ABP e tipos de satisfação & $\begin{array}{l}\text { Adotou-seo Revised Ways of Coping } \\
\text { Questionnaire (RWCQ) para investigar os } \\
\text { componentes e determinantes de situações } \\
\text { estressantes. }\end{array}$ \\
\hline
\end{tabular}

Fonte: Elaborado pelos autores.

\section{Estudos incluídos}

Esta revisão contém somente artigos não randomizados, sendo seis transversais e quatro longitudinais (incluindo dois estudos prospectivos e dois caso-controle), totalizando um espaço amostral de 4.155 pacientes. Em relação à representação geográfica dos estudos incluídos, avaliamos estudos das Américas (quatro), da Europa (três) e da Ásia (três). Nenhum estudo da África e Oceania foi incluído. Informações adicionais sobre os estudos incluídos estão no Quadro1. Os anos de publicação variaram de 1998 a 2018, e todos os estudos foram publicados em língua inglesa.

\section{Estudos excluídos}

Dentre os artigos completos avaliados para elegibilidade, dez foram excluídos. Oito estudos não avaliavam a saúde mental do estudante. Um estudo era uma revisão de literatura. $\mathrm{O}$ último estudo excluído não avaliava a saúde mental dos estudantes de Medicina submetidos ao método $\mathrm{ABP}$, e sim ao método tradicional. Os estudos estavam em língua portuguesa ou em língua inglesa e foram devidamente traduzidos antes da efetiva exclusão.

\section{Variáveis de saúde mental investigadas}

As variáveis de saúde mental analisadas pelos estudos foram: gostar do curso, insegurança por um currículo confuso, preocupação com o aprendizado médico no âmbito do desempenho e ambiente, competição relacionada ao método, preocupação com a insuficiência da educação, falta de apoio e feedback docente, falta de tempo para estudos curriculares e extracurriculares, isolamento social, expectativa com o curso, burnout, satisfação com o método $\mathrm{ABP}$, depressão, ansiedade, estresse, empatia, abertura à espiritualidade, bem-estar e qualidade de vida.
Resultados dos estudos incluídos na revisão sistemática

\begin{tabular}{|c|c|}
\hline \multicolumn{2}{|c|}{$\begin{array}{l}\text { Quadro } 2 \\
\text { Resultados finais dos artigos após a coleta e análise dos dados }(\mathrm{n}=10)\end{array}$} \\
\hline Primeiro autor & Resultados dos estudos no âmbito de saúde mental \\
\hline Lewis et al. ${ }^{23}$ & $\begin{array}{l}\text { Os alunos submetidos ao método } \mathrm{ABP} \text {, em } \\
\text { detrimento dos alunos submetidos ao ensino } \\
\text { tradicional, relataram que gostam do curso e não } \\
\text { sabem o que o corpo decente espera deles. Além } \\
\text { disso, apontaram os seguintes aspectos: presença } \\
\text { de um currículo pouco claro, preocupação em não } \\
\text { conseguirem dominar todo o conteúdo médico, } \\
\text { percepção de que a educação recebida não prepara } \\
\text { para a atividade clínica, falta de tempo para estudar } \\
\text { outros assuntos acadêmicos e praticar outras } \\
\text { atividades acadêmicas, falta de tempo para atividades } \\
\text { extracurriculares, falta de apoio dos colegas e } \\
\text { decepção com as expectativas criadas pelo curso. }\end{array}$ \\
\hline Barbosa et al. $^{22}$ & $\begin{array}{l}\text { Os alunos submetidos à metodologia } \mathrm{ABP} \text { foram } \\
\text { diagnosticados com uma taxa de burnout de } 12 \% \text { da } \\
\text { amostra }(\mathrm{n}=399) \text {, classificada como baixa. }\end{array}$ \\
\hline Bahar-ÖzvariŞetal. $^{16}$ & $\begin{array}{l}\text { Os alunos que foram submetidos ao método ABP, } \\
\text { com a aplicação de aprendizagem cooperativa, } \\
\text { relataram que os membros do grupo instigavam } \\
\text { uns aos outros a dominar o conteúdo e também } \\
\text { se ajudavam. Além disso, mencionaram que foi } \\
\text { agradável trabalhar em grupo. }\end{array}$ \\
\hline
\end{tabular}

Continua...

\begin{tabular}{l|l}
\hline REVISTA BRASILEIRA DE EDUCAÇÃO MÉDICA \\
\hline $44(4):$ el15; 2020
\end{tabular} 


\begin{tabular}{|c|c|}
\hline & $\begin{array}{c}\text { Quadro } 2 \\
\text { Continuação. }\end{array}$ \\
\hline Primeiro autor & Resultados dos estudos no âmbito de saúde mental \\
\hline Lucchetti et al. ${ }^{24}$ & $\begin{array}{l}\text { Os alunos brasileiros submetidos ao método } \mathrm{ABP} \text {, } \\
\text { em comparação com os alunos norte-americanos } \\
\text { submetidos ao mesmo método, relataram } \\
\text { significativamente mais depressão, maiores níveis de } \\
\text { estresse, menor bem-estar, maior exaustão e menor } \\
\text { qualidade de vida ambiental. }\end{array}$ \\
\hline Trappler $^{17}$ & $\begin{array}{l}\text { Os alunos submetidos à } \mathrm{ABP} \text { relataram que o } \\
\text { método foi bastante eficaz nos seus objetivos. No } \\
\text { entanto, mencionaram, majoritariamente, que não o } \\
\text { preferem. }\end{array}$ \\
\hline Yan et al. ${ }^{18}$ & $\begin{array}{l}\text { Os estudantes tiveram contato com a } \mathrm{ABP} \text { em } \\
\text { apenas um módulo, e a maioria concordou que o } \\
\text { método proporcionou maior satisfação, visto que } \\
\text { permitiu o desenvolvimento de algumas habilidades } \\
\text { autodirecionadas, bem como a possibilidade de lidar } \\
\text { melhor com o estresse. }\end{array}$ \\
\hline Holen et al. ${ }^{21}$ & $\begin{array}{l}\text { Há uma relação entre personalidade e satisfação } \\
\text { com a ABP, e os estudantes menos sociaveis, mais } \\
\text { introspectivos e timidos são os que apresentam } \\
\text { menor grau de satisfação. }\end{array}$ \\
\hline Jiménez-Mejíaset al. ${ }^{19}$ & $\begin{array}{l}\text { O método ABP promove não só uma maior } \\
\text { satisfação dos estudantes, mas também maior } \\
\text { desempenho. }\end{array}$ \\
\hline Alimogluet al. ${ }^{20}$ & $\begin{array}{l}\text { Os estudantes que desenvolveram estratégias de } \\
\text { enfrentamento com foco nos problemas (maioria } \\
\text { dos estudantes) foram os mais satisfeitos com as } \\
\text { atividades relacionadas à ABP (práticas), bem como } \\
\text { obtiveramos melhores resultados nas avaliações. }\end{array}$ \\
\hline Schauber et al. ${ }^{25}$ & $\begin{array}{l}\text { A interação entre as emoções dos estudantes, as } \\
\text { avaliações, as percepções e os resultados depende do } \\
\text { contexto educacional. }\end{array}$ \\
\hline
\end{tabular}

Fonte: Elaborado pelos autores.
Risco de viés nos estudos incluídos

A Figura 2 mostra as características dos estudos incluídos e orisco de viés, e a Figura 3 apresentao sumário de risco de viés: julgamentos do autor da revisão sobre cada item de risco de viés nos estudos incluídos.

De acordo com a ferramenta Robins-I, na Figura 3, três dos estudos Bahar-ÖzvariŞ et al. ${ }^{16}$, Trappler ${ }^{17}$ e Yan et al. ${ }^{18}$-que avaliaram a satisfação tiveram um risco crítico de viés, outros três estudos - Alimogluet al. ${ }^{20}$, Holenet al. ${ }^{21}$ e Lewis et al. ${ }^{23}$-tiveram um risco moderado de viés, com exceção do estudo de Jiménez-Mejíaset al. ${ }^{19}$ que apresentou risco grave. Os dois estudos - Barbosa et al. ${ }^{22}$ e Lucchettiet al. ${ }^{24}$ - que avaliaram burnoute distúrbios psicológicos tiveram um grave risco de viés. Um dos estudos Schauberet al. ${ }^{25}$-que avaliou o apoio social e ambiente de aprendizagem apresentou risco crítico de viés.

\section{DISCUSSÃO}

Dentre os dezestudos finais analisados, Bahar-Özvariş et al. ${ }^{16}$, Trappler $^{17}$, Yan et al. $^{18}$, Jiménez-Mejíaset al. ${ }^{19}$ e Alimogluet al. ${ }^{20}$ relacionaram a ABP com um melhor desempenho dos estudantes, ou seja, o método foi capaz de promover o desenvolvimento de habilidades auto direcionadas, bem como uma aprendizagem cooperativa. Nesse sentido, alguns estudos relataram resultados melhores em avaliações de grupos sob a metodologia ABP em comparação com grupos submetidos ao método tradicional. Esse fator pode estar atrelado a um maior grau de satisfação em relação à $\mathrm{ABP}$, visto que algumas características relevantes para uma boa saúde mental podem ser adquiridas no decorrer do método, como a autoconfiança, a persistência, a cooperação e a capacidade de lidar melhor com o estresse.

É importante destacar também o fato de as características pessoais interferirem na satisfação do estudante em relação à ABP. Nessa perspectiva, Alimogluet al. ${ }^{20} \mathrm{e}$ Holenet al. ${ }^{21}$ demonstraram que tanto alunos capazes de desenvolver estratégias de enfrentamento eficazes com foco seus problemas quanto aqueles mais extrovertidos são os mais satisfeitos com o método. Isso decorre do fato de haver uma exposição constante desses estudantes, reforçada por sessões tutoriais, seminários e maior contato com a prática ainda nos primeiros semestres do curso. Desse modo, a personalidade é uma variável capaz de alterar a percepção acerca

\section{Figura 2}

Figura de risco de viés: julgamentos dos autores sobre cada item de risco de viés nos estudos incluídos - em porcentagem

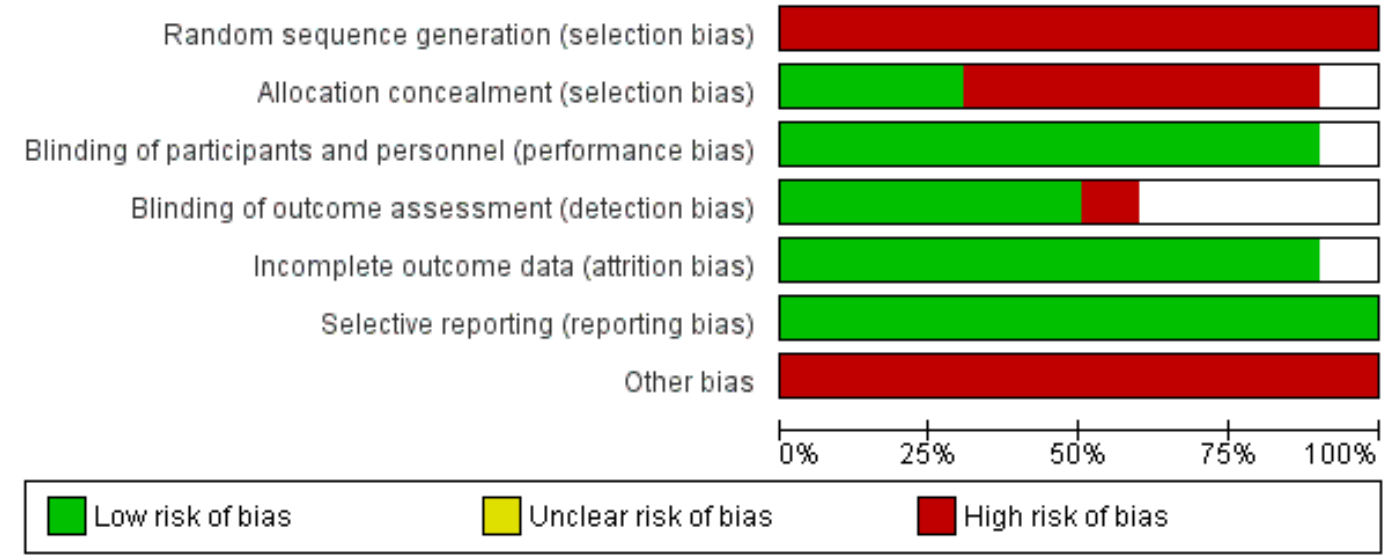

Fonte: Elaborada pelos autores

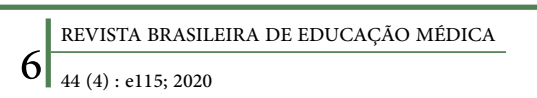




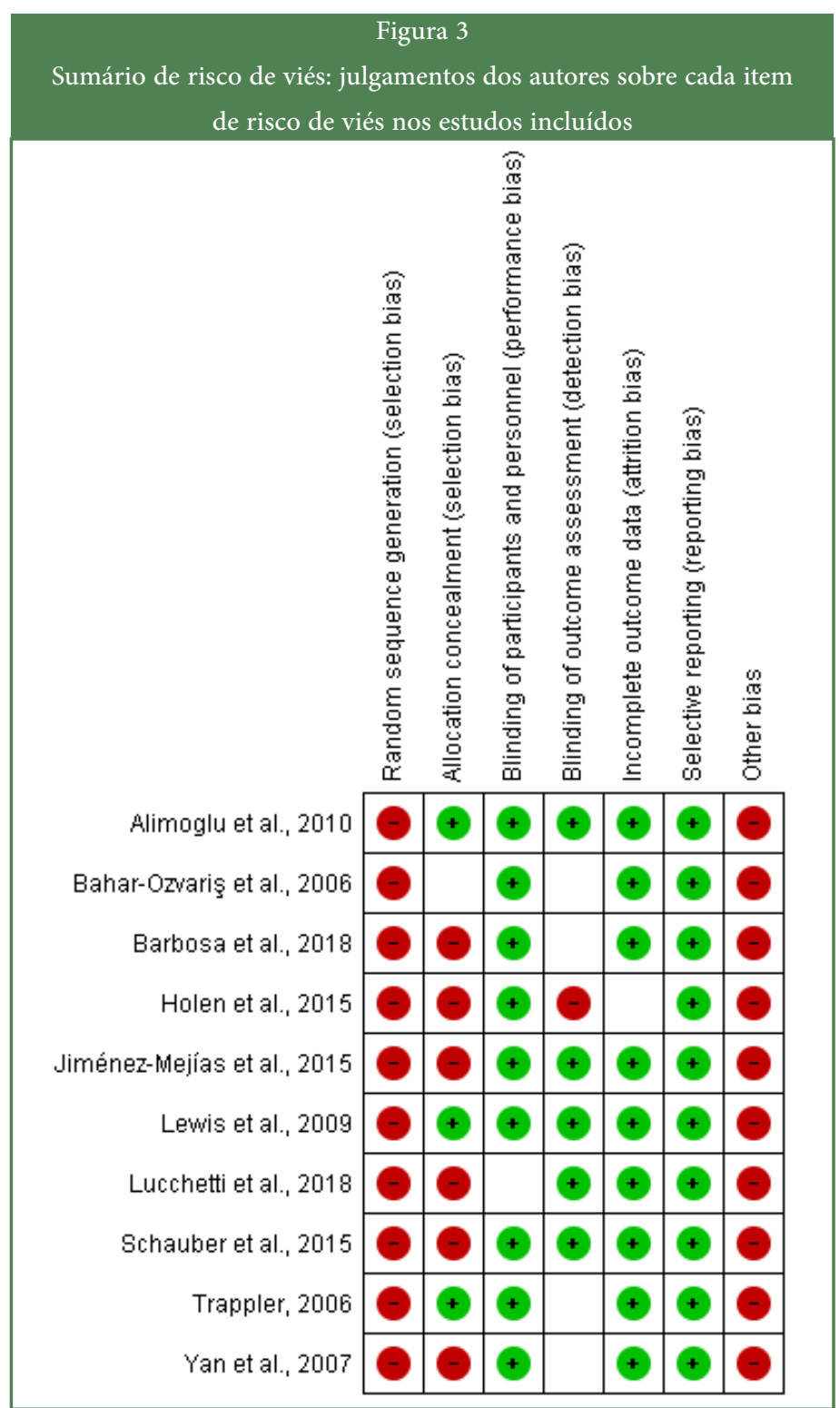

Fonte: Elaborada pelos autores da metodologia, bem como contribuir para uma maior probabilidade do adoecimento mental em estudantes tímidos e mais introvertidos.

Ademais, Barbosa et al. ${ }^{22}$ analisarama taxa de burnout e constataram um índice considerado baixo: $12 \%$ da amostra analisada. Por sua vez, Trappler $^{17}$ observou que a maioria dos participantes mostrou maior preferência pelo método tradicional. Isso pode estar relacionado a algumas percepções individuais sobre o método relatadas por Lewis et al. ${ }^{23}$ : não saber o que o corpo docente espera dos estudantes, presença de um currículo pouco claro, preocupação em não conseguir dominar todo o conteúdo médico, perceber que a educação recebida não prepara para a atividade clínica, falta de tempo para estudar outros assuntos acadêmicos e praticar outras atividades acadêmicas, falta de tempo para atividades extracurriculares, falta de apoio dos colegas e decepção com as expectativas criadas pelo curso, entre outras. Por fim, o estudo de Lucchetti et al. ${ }^{24}$, que comparou os alunos brasileiros de Medicina com os norte-americanos, revelou que os brasileiros têm significativamente mais depressão, maiores níveis de estresse, menor bem-estar, maior exaustão e menor qualidade de vida ambiental. Isso pode ser explicado por alguns fatores: diferenças culturais (pessoas latinas tendem a ser mais emotivas), imaturidade (possibilidade, no Brasil, de ingresso de estudantes muito jovens nas universidades), qualidade de vida (os Estados Unidos superam o Brasil nesse quesito) e tamanho das classes (no Brasil, há, em geral, muitos alunos nas salas de aula $)^{24}$.

Os artigos selecionados para a revisão sistemática convergem no sentido de que todos admitem que os estudantes de medicina submetidos ao método de ensino ABP apresentam estressores específicos desse tipo de metodologia, que, dependendo do estressor, é superior, ou não, à metodologia tradicional; no entanto, é fato que, em ambos, há diversos estressores que prejudicam a saúde mental dos estudantes. Desse modo, a metodologia da APB requer constante participação e exposição do discente, fato que promove a insatisfação dos alunos tímidos ${ }^{20,21}$. Ademais, a falta de tempo para o lazer, a falta de estrutura física, a solidão e a insegurança quanto ao método são fatores agravantes para a saúde mental dos alunos ${ }^{23}$. Como consequência disso, há a prevalência de sintomas de ansiedade e depressão em estudantes de Medicina, fatores que promovem uma redução no rendimento da aprendizagem e nas tarefas cotidianas e a

\begin{tabular}{|c|c|c|c|c|c|c|c|}
\hline \multicolumn{8}{|c|}{ Quadro 3} \\
\hline Autor (ano) & Confundimento & $\begin{array}{c}\text { Seleção dos } \\
\text { participantes }\end{array}$ & $\begin{array}{l}\text { Aferição da } \\
\text { intervenção }\end{array}$ & $\begin{array}{l}\text { Não recebimento da } \\
\text { intervenção atribuída }\end{array}$ & Perdas & $\begin{array}{l}\text { Aferição dos } \\
\text { desfechos }\end{array}$ & $\begin{array}{l}\text { Relato seletivo } \\
\text { dos desfechos }\end{array}$ \\
\hline Lewis et al., 2009 & Risco moderado & Risco moderado & Baixo risco & Baixo risco & Sem informação & Risco moderado & Baixo risco \\
\hline Barbosa et al., 2018 & Grave risco & Risco moderado & Baixo risco & Baixo risco & Sem informação & Risco moderado & Baixo risco \\
\hline Bahar-ÖzvariŞ et al., 2006 & Grave risco & Risco moderado & Risco moderado & Baixo risco & Sem informação & Baixo risco & Risco crítico \\
\hline Lucchetti et al.,2018 & Grave risco & Grave risco & Baixo risco & Baixo risco & Sem informação & Risco moderado & Baixo risco \\
\hline Trappler, 2006 & Risco crítico & Risco moderado & Risco moderado & Baixo risco & Sem informação & Risco moderado & Baixo risco \\
\hline Yan et al., 2017 & Risco crítico & Risco moderado & Risco moderado & Baixo risco & Baixo risco & Risco moderado & Risco moderado \\
\hline Holen et al., 2015 & Risco moderado & Risco moderado & Risco moderado & Baixo risco & Sem informação & Baixo risco & Baixo risco \\
\hline Jiménez-Mejías et al., 2015 & Grave risco & Risco moderado & Risco moderado & Baixo risco & Sem informação & Baixo risco & Risco moderado \\
\hline Alimoglu et al.,2011 & Risco moderado & Risco moderado & Risco moderado & Baixo risco & Risco moderado & Risco moderado & Baixo risco \\
\hline Schauber et al., 2015 & Risco crítico & Risco moderado & Baixo risco & Baixo risco & Risco moderado & Risco moderado & Baixo risco \\
\hline
\end{tabular}

Fonte: Elaborado pelos autores.

\begin{tabular}{l|l}
7 & REVISTA BRASILEIRA DE EDUCAÇÃO MÉDICA \\
\hline 44 (4) : e115; 2020
\end{tabular} 
ocorrência de baixa autoestima e insegurança ${ }^{24}$.

De forma geral, os achados da presente revisão sistemática mostraram-se ambíguos, ora favorecendo a $\mathrm{ABP}$, em detrimento do modelo tradicional, ora o contrário. Além disso, constataram-se prejuízos mentais e fisiológicos pela deteriorada saúde mental que esses estudantes apresentam. Sobre as limitações, entende-se que a estratégia de inclusão não investigou artigos em todos os bancos de dados existentes. Além disso, os estudos revisados são de natureza observacional, o que já aumenta exponencialmente alguns riscos de viés, como o viés de confundimento. Por fim, sugerem-se pesquisas de estudo prospectivo longitudinal, com cegamento dos avaliadores, análise multivariável, considerando possíveis fatores limitantes, amostragem relevante e intervenção sistematizada baseada na literatura preexistente.

\section{CONCLUSÃO}

Esta revisão sistemática, pautada no método Cochrane, objetivou relacionar três fatores: estudantes de Medicina, saúde mental e ABP. Os resultados obtidos são importantes porque podem ser utilizados para definir diretrizes para o curso de Medicina, com o objetivo de melhorar a formação do profissional e, por conseguinte, a sua atuação no mercado de trabalho. De forma geral, os achados do presente estudo mostraram-se predominantemente em favor da $\mathrm{ABP}$ como a metodologia que promove melhor resultado no que concerne à saúde mental, o que não significa, contudo, a inexistência de transtornos mentais entre os participantes do modelo ABP, mas, sim, uma menor taxa em comparação com o modelo tradicional. Os artigos selecionados para a revisão sistemática convergem no sentido de que todos admitem que os estudantes de Medicina submetidos ao método de ensino ABP apresentam estressores específicos desse tipo de metodologia. No entanto, é fato que, em ambos, há diversos estressores que prejudicam a saúde mental dos estudantes.

\section{REFERÊNCIAS}

1. Campbell RJ. Dicionario de psiquiatria. 8th ed. Massachusetts: Jones e Bartlett;2008.

2. Gorestein C, Wang YP, Hungerbühler I. Instrumentos de avaliação em saúde mental. Porto Alegre: Artmed;2016.

3. Andreasen NC. Introdução à Psiquiatria. Porto Alegre: Artmed, 2009.

4. da Silveira ALP, Colleta TCD, Ono HRB, Woitas LR, Soares $\mathrm{SH}$, Andrade VLA, et al. Síndrome de burnout:consequências e implicações de uma realidade cada vez mais prevalente na vida dos profissionais de saúde. RevBrasMedTrab.2016;14(3):275-84.

5. Meireles MAC, Fernandes CCP, Silva LS. Novas diretrizes curriculares nacionais e a formação médica: expectativas dos discentes do primeiro ano do curso de Medicina de uma instituição de ensino superior. Rev Bras Educ Med. 2019;43(2):67-78.

6. Santos FS, Maia CRC, Faedo FC, Gomes GPC, Nunes ME, de Oliveira MVM. Estresse em estudantes de cursos preparatórios e de graduação em medicina. Rev Bras Educ Med. 2017;41(2):194-200.

7. Pereira RC, de Oliveira AL, Viana HJ, Lima ARS, de Alencar MP. Metodologias ativas ou convencionais para o desenvolvimento de trabalhos de conclusão de curso? Uma análise da percepção de alunos do curso de Administração. RevMult Psic. 2018;12(41):371-89 [acesso em1 ${ }^{\circ}$ out 2019]. Disponível em:https://idonline.emnuvens.com.br/id/ article/view/1224.
8. Brasil. Ministério da Educação. Conselho Nacional de Educação. Câmara de Educação Superior Resolução CNE/CES n ${ }^{\circ} 3$ de 20 de junho de 2014. Institui Diretrizes Curriculares Nacionais do Curso de Graduação em Medicina.Diário Oficial da União,Brasília;23 jun2014; Seção 1, p. 8.

9. Lopes RM, Silva Filho MV, Marsden M, Alves NG. Aprendizagem baseada em problemas: uma experiência no ensino de química toxicologica. Quim Nova. 2011;34(7):1275-80.

10. Dias-Lima A, Silva MC, Ribeiro LCV, Bendicho MT, Guedes HTV, Lemaire DC. Avaliação, ensinagem e metodologias ativas: uma experiência vivenciada no componente curricular mecanismos de agressão e de defesa no curso de medicina da Universidade do Estado da Bahia, Brasil. Rev Bras Educ Med. 2019;43(2):216-24.

11. Monteiro CFS, Freitas JFM, Ribeiro AAP. Estresse no cotidiano acadêmico: o olhar dos alunos de Enfermagem da Universidade Federal do Piauí. Esc Anna Nery Rev Enferm. 2007;11(1):66-72.

12. Nogueira MJ, Sequeira C. A satisfação com a vida académica: relação com bem-estar e distress psicológico. Rev Port Enferm Saúde Ment. 2018;(esp 6):71-6.

13. Garro IMB, Camillo SDO, Nóbrega MDPSDS. Depressão em graduandos de enfermagem. Acta Paul Enferm.2006; 19(2):162-7.

14. Costa KMV, Sousa KRSS, Formiga PA, Silva WS, Bezerra EBN. Ansiedade em universitários na área da saúde. II Congresso Brasileiro de Ciências da Saúde; Campina Grande:Univale.2017.

15. Moher D, Liberati A, Tetzlaff J, Altman DG, The PRISMA Group. Preferred Reporting Items for Systematic Reviews and Meta-Analyses: The PRISMA Statement. PLoS Med. 2009;6(7): e1000097. doi: 10.1371/ journal.pmed.1000097.

16. Bahar-Özvariş Ş, Çetin FÇ, Turan S, Peters AS. Cooperative learning: anew application of problem-based learning in mental health training. Med Teach. 2006;28(6):553-7.

17. Trappler B. Integrated problem-based learning in the neuroscience curriculum - The SUNY Downstate experience. BMC Med Educ. 2006;6(47):1-7.

18. Yan Q, Ma L, Zhu L, Zhang W. Learning effectiveness and satisfaction of international medical students: introducing a hybrid - PBL curriculum in biochemistry. Biochem Mol Biol Educ. 2017;45(4):336-42.

19. Jiménez-Mejías E, Amezcua-Prieto C, Martínez-Ruiz V, Olvera-Porcel MC, Jiménez-Moleón JJ, Claret PL. Medical student's satisfaction and academic performance with problem-based learning in practicebased exercises for epidemiology and health demographics. Innov Educ Teach Int. 2015;52(5):510-21.

20. Alimoglu MK, Gurpinar E, Mamakli S, Aktekin M. Ways of coping as predictors of satisfaction with curriculum and academic success in medical school. Am J Physiol -Adv Physiol Educ. 2011;35:33-8.

21. Holen A, Manandhar K, Pant DS, Karmacharya BM, Olson LM, Koju $\mathrm{R}$, et al. Medical students' preferences for problem-based learning in relation to culture and personality: amulticultural study. Int J Med Educ. 2015;6:84-92.

22. Barbosa ML, Ferreira BLR, Vargas TN, da Silva GM, Nardi AE, Machado S,et al. Burnout prevalence and associated factors among Brazilian medical students. Clin Pract Epidemiol Ment Heal.2018;14:188-95.

23. Lewis $\mathrm{AD}$, Menezes $\mathrm{DAB}$, McDermott HE,Hibbert LJ, Brennan SL, Ross EE, et al. A comparison of course-related stressors in 
undergraduate problem-based learning (PBL) versus non-PBL medical programmes. BMC Med Educ. 2009;9(60):1-8.

24. Lucchetti G, Damiano RF, DiLalla LF,Lucchetti ALG, Moutinho ILD,da Silva Ezequiel O, et al. Cross-cultural differences in mental health, quality of life, empathy, and burnout between US and Brazilian medical students. Acad Psychiatry. 2018;42(1):62-7.

25. Schauber SK, Hecht M, Nouns ZM, Kuhlmey A, Dettmer S. The role of environmental and individual characteristics in the development of student achievement: a comparison between a traditional and a problembased-learning curriculum. Adv Heal Sci Educ. 2015;47:777-80.

\section{CONTRIBUICCÃO DOS AUTORES}

Carlos Emanoel Chaves da Silva e Alef Oliveira do Nascimento foram responsáveis pela conceituação do trabalho, curadoria de dados, investigação, metodologia e redação (rascunho original). Israel Junior Borges do Nascimento, Mayra Hermínia Simões Hamad Farias do
Couto e Luiz Euclides Coelho de Souza Filho foram responsáveis pela conceituação do trabalho, curadoria de dados, análise formal, investigação, metodologia, redação (rascunho original, revisão e edição), participou da revisão, edição (suporte) do artigo e da análise estatística. Katiane da Costa Cunha foi responsável pela conceituação do trabalho, curadoria de dados, análise formal, investigação, metodologia, redação (rascunho original, revisão e edição), participou da revisão, edição (suporte) do artigo, da análise estatística, administração e supervisão do projeto.

\section{CONFLITO DE INTERESSES}

Os autores declaram não haver conflito de interesses neste estudo.

\section{ENDEREÇO PARA CORRESPONDÊNCIA}

Mayra Hermínia Simões Hamad Farias do Couto. Avenida Conselheiro Furtado, 2312, apartamento 302, Bairro Cremação, Belém, PA, Brasil. CEP: 66040-105.

E-mail: Mayrahamad@gmail.com 